\title{
Machine Learning to Analyze Single-Case Data: A Replication and Extension
}

\author{
Marc J. Lanovaz ${ }^{1,2}$ \\ ${ }^{1}$ École de psychoéducation, Université de Montréal \\ ${ }^{2}$ Centre de recherche de l'Institut universitaire en santé mentale de Montréal
}

\section{Author Note}

This study was supported in part by a salary award from the Fonds de recherche du Québec - Santé (\#269462) to the first author.

Correspondence concerning this article should be addressed to Marc J. Lanovaz, École de psychoéducation, Université de Montréal, C.P. 6128, succursale Centre-Ville, Montreal, QC, Canada, H3C 3J7. Email: marc.lanovaz@umontreal.ca, Phone: 1 514-343-6111 \#81774 


\begin{abstract}
Recently, Lanovaz et al. (2020) have found that machine learning algorithms may adequately control for Type I error rate and power when analyzing single-case graphs. However, the study limited most of its analyses to simulated datasets. To replicate and extend this study, we applied the four machine learning models developed by Lanovaz et al. (2020) to a previously published nonsimulated dataset. On average, the four models produced lower proportions of false positives than well-established methods to analyze AB graphs (i.e., the dual-criteria and conservative dual-criteria methods). These results support the use of machine learning to analyze single-case graphs, but further replication by an independent research team using educational and clinical data remains necessary.
\end{abstract}

Keywords: AB design, artificial intelligence, false positives, machine learning, singlecase design 


\section{Machine Learning to Analyze Single-Case Data: A Replication and Extension}

Several studies have questioned the reliability of visual analysis for identifying behavior changes on single-case graphs (DeProspero \& Cohen, 1979; Ninci et al., 2015; Wolfe et al., 2016, 2018). To address this issue, researchers have proposed different methods to more objectively analyze single-case data (Fisher et al., 2003; Krueger et al., 2013; Lanovaz et al., 2020; Manolov \& Vannest, 2020). Notably, Fisher et al. (2003) developed the dual-criteria and conservative dual-criteria methods, which have been the topic of a growing number of studies examining their validity (Falligant et al., 2020; Lanovaz et al., 2017, 2020; Wolfe et al., 2018). While researchers have shown that these methods may adequately control Type I error rates (e.g. Falligant et al., 2020; Lanovaz et al., 2017), studies have also noted that their power could benefit from improvements (Fisher et al., 2003; Manolov \& Vannest, 2020).

Recently, some researchers have proposed using machine learning to analyze single-case graphs (Lanovaz et al., 2020). Machine learning involves providing data to a computer algorithm that will learn to recognize patterns. The algorithm trains a model using input data, which is then taught to make predictions (output data). To test the accuracy of the trained model, researchers examine the model's predictions on novel, untrained data (i.e., generalization). In behavior analytic terms, the algorithm is the teaching procedure, the model is the learner, and the predictions are the responses. The input data, or features, are the discriminative stimuli provided to the algorithm whereas the labels are the correct responses. In the analysis of single-case graphs, the algorithms use the mean, standard deviation, slope and intercept of Phases A and B as input data, and the labels are whether the graphs show a clear change or not as recorded by an expert visual analyst. As such, the output, or prediction, is a binary variable indicating whether the graph shows a clear change or not. 
In a proof of concept, Lanovaz et al. (2020) trained models using four different algorithms to identify changes in single-case $\mathrm{AB}$ graphs. Their results showed that all models produced lower Type I error rates (less false positives) and higher power (less false negatives) than the dual-criteria method on simulated data. Furthermore, the models had higher agreement with visual analysts than the dual-criteria method on data extracted from theses and dissertations. However, the results must be replicated with nonsimulated datasets that were not used as part of the original training. Lanovaz et al. (2017) created such a dataset to measure false positives in nonsimulated data. This latter study only focused on the dual-criteria method as the machine learning models to analyze single-case graphs had not been developed yet. Therefore, the purpose of our study was to replicate and extend Lanovaz et al. $(2017,2020)$ by comparing proportions of false positives produced by models derived from machine learning in a nonsimulated dataset comprised of $\mathrm{AB}$ graphs with different phase lengths.

\section{Method}

\section{Dataset}

The current study used the same dataset as previously described by Lanovaz et al. (2017). The dataset contained 295 baseline phases extracted from 73 studies published in four peerreviewed journals. Each baseline had 6 to 20 points. A program written by the first author in Python split the baseline data to produce data series containing no less than six points and no more than 12 points in total. For example, a baseline phase with nine points could be divided into 10 data series: four series with six points (i.e., points 1 to 6,2 to 7,3 to 8 and 4 to 9), three series with seven points (i.e., points 1 to 7,2 to 8 and 3 to 9), two series with eight points (i.e., points 1 to 8 and 2 to 9 ), and one series with nine points (i.e., points 1 to 9). Finally, our program further divided each series into $\mathrm{AB}$ graphs by placing a phase change line at all locations that allowed 
the graph to have a minimum of three points and a maximum of six points per phase. For the prior series with nine points, we could locate the phase change line between the third and fourth points, the fourth and fifth points, the fifth and six points, and the sixth and seventh points. In total, these manipulations yielded 16,927 AB graphs for analysis.

\section{Analysis}

Our program used the models developed by Lanovaz et al. (2020) to analyze each AB graph. First, a Python function multiplied all values of the AB graph by -1 when the purpose of the intervention of the graph from which the baseline was extracted was to decrease the behavior. This same function then normalized the data by transforming each value of the AB graph to a $z$ score so that the mean of each graph was now 0 and the standard deviation was 1 . The final step involved the function extracting the eight features: mean of Phase A, mean of Phase B, standardized deviation of Phase A, standardized deviation of Phase B, intercept and slope of the least squared means line for Phase A, and intercept and slope of the least squared means line for Phase B. These eight features functioned as the input data that were provided to the models.

We tested the models derived from four machine learning algorithms: the stochastic gradient descent classifier, the support vector classifier, the random forest classifier, and the dense neural network. Describing these algorithms in detail goes beyond the scope of a replication study. Nonetheless, we included the descriptions of each algorithm provided by Lanovaz et al. (2020) as Supplementary Material to this article. Each model used the eight features for each graph to produce a binary output (0: no clear change, 1: clear change). Given that each AB graph only included baseline data, the analysis program categorized clear changes as false positives. That is, a false positive occurred when the model erroneously concluded that the $\mathrm{AB}$ graph showed a clear change. We computed the proportion of false positives by dividing 
the number of $\mathrm{AB}$ graphs showing a clear change with a specific number of points in Phases $\mathrm{A}$ and $\mathrm{B}$ by total number of $\mathrm{AB}$ graphs containing this same number points in Phases $\mathrm{A}$ and $\mathrm{B}$. The data and all code used for the current study are freely available at: https://osf.io/nr38m/.

\section{Results and Discussion}

Table 1 presents the proportions of false positives produced by the dual-criteria and conservative dual-criteria methods (from Lanovaz et al., 2017), and by the models derived from the four different machine learning algorithms. On average, the stochastic gradient descent classifier produced the lowest mean proportion of false positives $(M=.035)$ followed by the random forest classifier $(M=.043)$, the support vector classifier $(M=.046)$ and the dense neural network $(M=.047)$. In contrast, the dual-criteria $(M=.072)$ and the conservative dual-criteria $(M=.053)$ methods yielded the highest proportions of false positives. The best model, stochastic gradient descent, outperformed both dual-criteria methods whenever Phase A contained at least four points. The only conditions when at least one of the dual-criteria methods produced lower proportions of false positives was when the graphs had three points in Phase A while having four, five, or six points in Phase B.

Our results show that models derived from machine learning generally produced lower proportions of false positives than the dual-criteria and conservative dual-criteria methods on novel, untrained data. When using machine learning, the proportions of false positives decreased considerably when the number of points in Phase A increased, which was not the case for the dual-criteria and conservative dual-criteria methods (Falligant et al., 2020; Lanovaz et al., 2017). Considering that prior research has shown that machine learning also produced better power than the dual-criteria method, our results further support the adoption of machine learning for the analysis of AB graphs. Notably, the stochastic gradient descent classifier resulted in the lowest 
average proportion of false positives in the current study while producing the highest power (i.e., lowest average proportion of false negatives) in the Lanovaz et al. (2020) study. This result underlines the promising nature of this algorithm for analyzing single-case data and would merit further attention in future research.

Our replication has limitations that should be carefully considered. First, our study did not monitor, nor report false negatives. Measuring false negatives in nonsimulated data inextricably leads to circular reasoning, which is why we could not evaluate this measure as part of the current study. Second, Lanovaz et al. (2017) limited their dataset to published baseline data, which may have different properties than unpublished baseline data (Sham \& Smith, 2014). Future research should address this issue by analyzing data from dissertations and theses or using actual clinical and educational data (Falligant et al., 2020). Third, the unfamiliarity of behavior analysts with machine learning may hinder its adoption. To address these issues, Lanovaz et al. (2020) developed a tutorial for behavior analysts to replicate their procedures without the need for advanced knowledge in the domains of artificial intelligence and programming (see Tutorial.ipynb at https://osf.io/6wcty/). Furthermore, we developed an easy to use online calculator for behavior analysts (see https://labrl.shinyapps.io/MachineLearningABGraphs/), which analyzes $\mathrm{AB}$ graphs of any length using the stochastic gradient descent classifier. That said, an independent research team must replicate these results with a novel dataset to clearly establish the utility of using machine learning algorithms in research and practice. 


\section{References}

DeProspero, A., \& Cohen, S. (1979). Inconsistent visual analyses of intrasubject data. Journal of Applied Behavior Analysis, 12(4), 573-579. https://doi.org/10.1901/jaba.1979.12-573

Falligant, J. M., McNulty, M. K., Hausman, N. L., \& Rooker, G. W. (2020). Using dual-criteria methods to supplement visual inspection: Replication and extension. Journal of Applied Behavior Analysis. Advance online publication. https://doi.org/10.1002/jaba.665

Fisher, W. W., Kelley, M. E., \& Lomas, J. E. (2003). Visual aids and structured criteria for improving visual inspection and interpretation of single-case designs. Journal of Applied Behavior Analysis, 36(3), 387-406. https://doi.org/10.1901/jaba.2003.36-387

Krueger, T. K., Rapp, J. T., Ott, L. M., Lood, E. A., \& Novotny, M. A. (2013). Detecting false positives in A-B designs: Potential implications for practitioners. Behavior Modification, 37(5), 615-630. https://doi.org/10.1177/0145445512468754

Lanovaz, M. J., Giannakakos, A. R., \& Destras, O. (2020). Machine learning to analyze singlecase data: A proof of concept. Perspectives on Behavior Science. Advance online publication. https://doi.org/1.1007/s40614-020-00244-0

Lanovaz, M. J., Huxley, S. C., \& Dufour, M. M. (2017). Using the dual-criteria methods to supplement visual inspection: An analysis of nonsimulated data. Journal of Applied Behavior Analysis, 50(3), 662-667. https://doi.org/10.1002/jaba.394

Manolov, R., \& Vannest, K. J. (2020). A visual aid and objective rule encompassing the data features of visual analysis. Behavior Modification. Advance online publication. https://doi.org/10.1177/0145445519854323 
Ninci, J., Vannest, K. J., Willson, V., \& Zhang, N. (2015). Interrater agreement between visual analysts of single-case data: A meta-analysis. Behavior Modification, 39(4), 510-541. https://doi.org/10.1177/0145445515581327

Sham, E., \& Smith, T. (2014). Publication bias in studies of an applied behavior-analytic intervention: An initial analysis. Journal of Applied Behavior Analysis, 47(3), 663-678. https://doi.org/10.1002/jaba.146

Wolfe, K., Seaman, M. A., \& Drasgow, E. (2016). Interrater agreement on the visual analysis of individual tiers and functional relations in multiple baseline designs. Behavior Modification, 40(6), 852-873. https://doi.org/10.1177/0145445516644699

Wolfe, K., Seaman, M. A., Drasgow, E., \& Sherlock, P. (2018). An evaluation of the agreement between the conservative dual-criterion method and expert visual analysis. Journal of Applied Behavior Analysis, 51(2), 345-351. https://doi.org/10.1002/jaba.453 


\section{Table 1}

Proportion of False Positives Produced by Each Analysis Method

\begin{tabular}{|c|c|c|c|c|c|c|c|}
\hline \multicolumn{2}{|c|}{ Number of Points } & \multicolumn{6}{|c|}{ Proportion of False Positives } \\
\hline Phase A & Phase B & DC & $\mathrm{CDC}$ & SGD & SVC & $\begin{array}{r}\text { Random } \\
\text { Forest }\end{array}$ & DNN \\
\hline 3 & 3 & .115 & .087 & .083 & .072 & .077 & .100 \\
\hline 3 & 4 & .084 & .063 & .079 & .067 & .063 & .067 \\
\hline 3 & 5 & .056 & .044 & .066 & .071 & .056 & .061 \\
\hline 3 & 6 & .041 & .031 & .066 & .072 & .045 & .053 \\
\hline 4 & 3 & .121 & .091 & .038 & .033 & .053 & .066 \\
\hline 4 & 4 & .084 & .062 & .037 & .046 & .045 & .040 \\
\hline 4 & 5 & .055 & .038 & .034 & .041 & .039 & .038 \\
\hline 4 & 6 & .040 & .025 & .025 & .044 & .031 & .028 \\
\hline 5 & 3 & .122 & .093 & .025 & .049 & .051 & .071 \\
\hline 5 & 4 & .079 & .060 & .022 & .028 & .041 & .031 \\
\hline 5 & 5 & .048 & .035 & .018 & .033 & .028 & .032 \\
\hline 5 & 6 & .025 & .019 & .014 & .033 & .022 & .019 \\
\hline 6 & 3 & .121 & .087 & .017 & .074 & .052 & .075 \\
\hline 6 & 4 & .081 & .060 & .015 & .020 & .045 & .031 \\
\hline 6 & 5 & .042 & .036 & .015 & .021 & .030 & .026 \\
\hline 6 & 6 & .032 & .019 & .005 & .026 & .016 & .016 \\
\hline
\end{tabular}

Note. DC: dual-criteria method, CDC: conservative dual-criteria method, SGD: stochastic gradient descent, SVC: support vector classifier, DNN: dense neural network. We highlighted the lowest Type I error for each given combination of points in Phases A and B. 


\section{Stochastic Gradient Descent Classifiers}

Stochastic gradient descent in machine learning is analogous to shaping in behavior analysis. That is, the algorithm produces successive predictions (approximations) of the labels until these predictions best matches the true labels (terminal behavior). During training, stochastic gradient involves only keeping the model that produces the best accuracy while discarding the others (as differential reinforcement does with behavior). In mathematical terms, the algorithm a) multiplies the input (features) by weights (parameters), b) transforms the previous products using a function that produces predictions, and c) updates the weights by applying a correction related to the gradient of the error on the predictions for the next iteration (Witten, Frank, Hall, \& Pal, 2017). The algorithm runs in a loop and updates its parameters at the end of each iteration (i.e., one epoch) to minimize the error of the predictions. In our models, the function that produced the predictions was a logistic regression and we manipulated the magnitude of the correction applied to the gradient across each epoch (learning rate). If the stochastic gradient descent were to run indefinitely, the error would eventually reach zero (i.e., perfect prediction). On the other hand, running too many epochs may produce models that fail to generalize to novel (untrained) data (i.e., overfitting). To address this issue, we varied the number of epochs to select the model that produced the best generalization to the validation set and added a penalty hyperparameter, ElasticNet, which is designed to minimize overfitting. 


\section{Support Vector Classifiers}

A support vector classifier works by producing a hyperplane in a higher-dimensional space to split the dataset according to the categorization of the initial labels (Witten et al., 2017). The figure below shows a simple example of how a hyperplane may separate the data in two categories. The left graph depicts the values of two categories (i.e., closed point and opened points) with two features (i.e., $\mathrm{x} 1$ and $\mathrm{x} 2$ ). Clearly, a simple linear equation (i.e., a straight line) cannot efficiently separate the two categories. The support vector classifier uses a function to separate the data in a higher dimension (see right panel). Now, a plane can separate the data depicted into two categories: the closed points are below the plane while the opened points are above. When this separation is conducted in higher dimensions (as in the case when having multiple features), we refer to these planes as hyperplanes. Support vector classifiers attempt to maximum the distance between the hyperplane and the data in each category. One hyperparameter for support vector classifiers is the kernel, which is a set of functions that transforms the data to allow separation. The radial basis function kernel, which we used as part of the current study, also has two hyperparameters: the $\mathrm{C}$ penalty term and gamma. The $\mathrm{C}$ penalty term modifies the margin of the separation of the hyperplane whereas gamma influences the weight of a single training exemplar. Both hyperparameters are manipulated together to minimize overfitting.
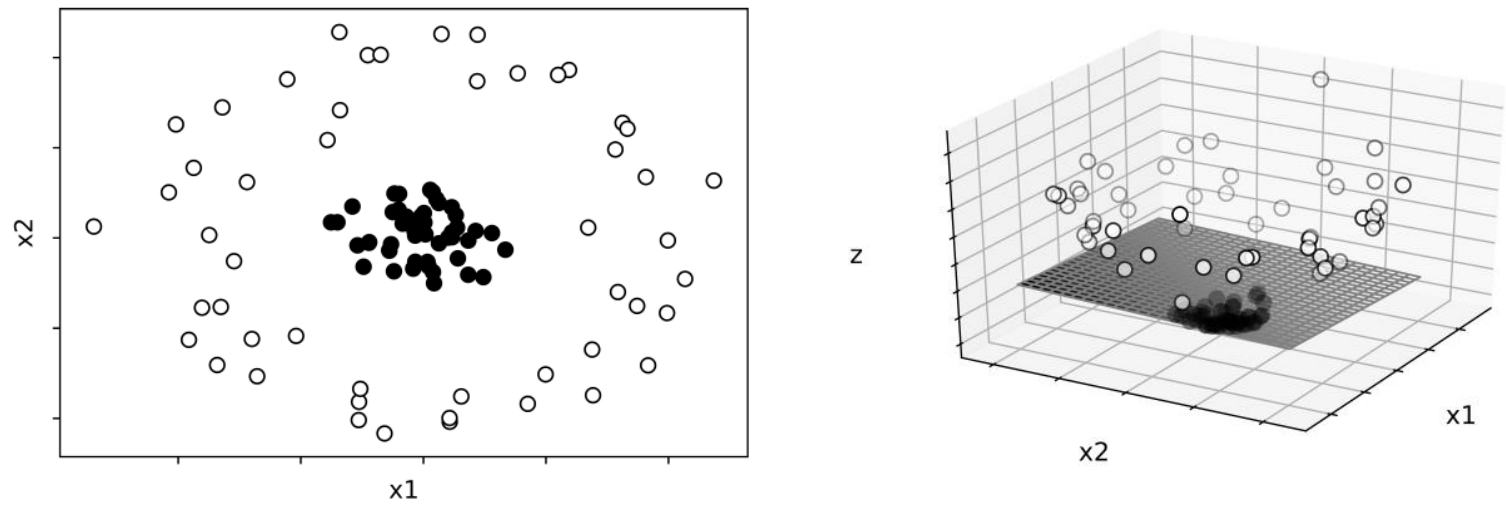

Figure legend. The left panel shows a two-dimensional graph representing two features: $x 1$ and $\mathrm{x} 2$. Closed points represent one category and opened points a different category. The right panel depicts the addition of a higher dimension ( $\mathrm{z}$ ) and a linear plan that separates the two categories. 


\section{Random Forest Classifiers}

Random forests are made up of a series of decision trees that are constructed during training (Breiman, 2001). As an example, assume that you are conducting discrete trial instruction with a child with autism and that you consider that the child has mastered a target when she responds correctly on more than $90 \%$ of trials for two consecutive sessions. The output (y) is whether the child has mastered the target $(y=1)$ or has not mastered the target $(y=0)$. The model has two features: $x 1$ represents the percentage of correct responding of the child on the next-to-last session and $\mathrm{x} 2$ the same measure on the last session. The figure below depicts the process as a decision tree. Random forests are similar except that the trees are automatically produced by algorithms containing a randomizing component (and not by some conceptual logic). Random forests create decision trees by taking a random sample with replacement of examples from the training set, selecting randomly permutated features at each decision node, and choosing the feature that produces the most homogeneous groups following the split. Then, the algorithm selects a new group of examples to sample a new tree and the process repeats itself. The algorithm produces many independent trees, which are referred to as a forest. The final classification is either the average prediction of all trees in the forest or the prediction that occurs most often. When developing a random forest classifier model, the number of trees in the forest are a hyperparameter known as estimators.

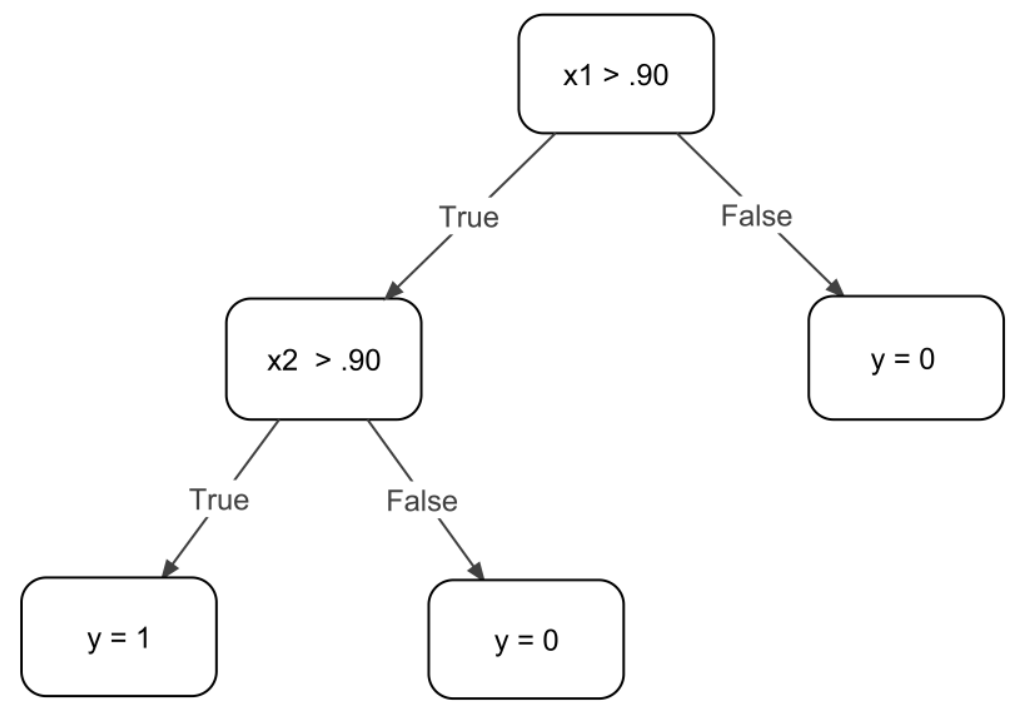

Figure legend. A decision tree where the percentage of correct responding in the next-to-last $(\mathrm{x} 1)$ and last $(\mathrm{x} 2)$ sessions are used to decide whether a concept is mastered $(\mathrm{y}=1)$ or not mastered $(y=0)$. 


\section{Dense Neural Networks}

Dense neural networks are artificial neural networks in which neurons are arranged in fullyconnected layers. In the context of machine learning, a neuron is a decision-making unit that receives some input and transforms it into some output using an activation function (Goodfellow, Bengio \& Courville, 2016). The figure below shows an example of a dense neural network. A dense neural network contains an input layer (i.e., the features), hidden layers with neurons, and an output layer (i.e., prediction). The hidden layers transform the data received from the input (or from other hidden layers) before outputting them to the next layer in the network. These hidden layers allow the algorithm to model complex relationships between features in the dataset. In a dense neural network, each neuron in a layer is connected to and receives input from every neuron in the layer preceding it. The activation function is a hyperparameter that produces a nonlinear transformation of the data between the layers and prior to producing the final output. During each epoch, the parameters of the model (i.e., weights) are updated by the loss function that computes the error and retropropagates it by an amount proportional to the learning rate (akin to a stochastic gradient descent with many layers of weights). Both the loss function and learning rate optimizer are hyperparameters. Our dense neural network models also included an early stopping hyperparameter, which instructs the network at which epoch it should stop training.

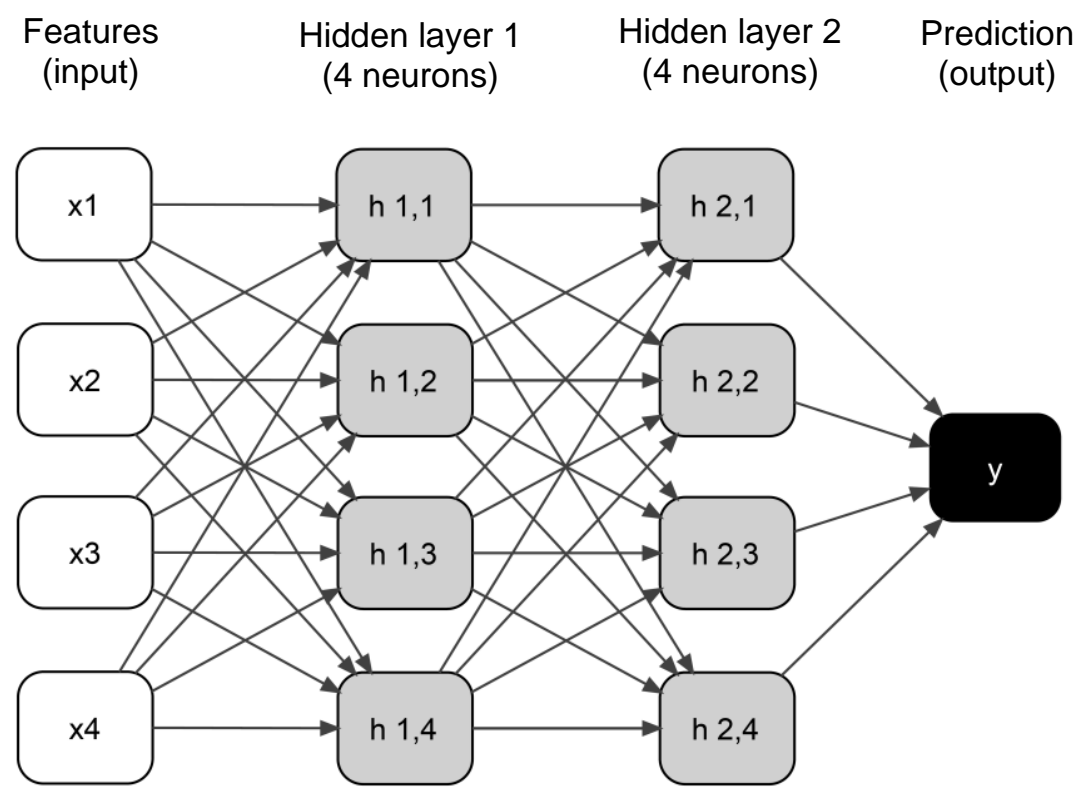

Figure legend. Dense neural network with four features, two hidden layers with four neurons each and a prediction. 